\title{
Influence of Blade Lean on Performance and Shock Wave/Tip Leakage Flow Interaction in a Transonic Compressor Rotor
}

\author{
Z. Cao, X. Zhang ${ }^{\dagger}$, Y. Liang and B. Liu \\ School of Power and Energy, Northwestern Polytechnical University, Xi'an 710072, China
}

†Corresponding Author Email: zxzxlchx@163.com

(Received March 3, 2021; accepted August 8, 2021)

\begin{abstract}
Blade lean has been extensively used in axial compressor stators to control flow separations, but its influence mechanism on transonic compressor rotors remains to be revealed. The aim of this study is to numerically explore the influence of blade lean on the performance and shock wave/tip leakage flow interaction in a transonic compressor rotor. The effects of leaned pattern (positively lean and negatively lean), leaned angle and leaned height were studied. Results showed that, compared with baseline configuration, the efficiency and total pressure ratio of the entire constant rotating speed line of positively leaned rotor were both decreased. The absolute value of peak efficiency was reduced by as much as $4.34 \%$ at $20^{\circ}$ lean angle, whereas the maximum reduction of peak total pressure ratio was 0.1 at $20^{\circ}$ lean angle. The tip leakage flow streamlines of baseline transonic rotor can be divided into two parts, i.e., the primary vortex and secondary vortex which arises after the shock. Due to shock/tip leakage vortex interaction, the primary vortex enlarged and low-momentum region showed up after the shock; under near stall (NS) condition, tip leakage vortex breakdown occurred after interacting with shock. As positively leaned angle increased, the shock and the shock/tip leakage vortex interaction point moved upstream. In addition, the phenomenon of tip leakage vortex breakdown was enhanced. For negatively leaned rotors, as negatively leaned angle increased, the peak efficiency and total pressure ratio showed a tendency of first increasing and then decreasing. At $5^{\circ}$ leaned angle, the peak efficiency was increased by $0.8 \%$ at most, and the maximum increment of total pressure ratio was 0.05 at $5^{\circ}$ leaned angle. Besides, the loading of blade tip reduced and the loading moved toward trailing edge, resulting in the downstream movements of primary vortex, shock front and shock/tip leakage vortex interaction location. The results may help to improve the near tip flow field of transonic compressor rotor with leaned blade technology.
\end{abstract}

Keywords: Blade lean; Tip leakage vortex; Shock/Tip leakage flow interaction; Transonic compressor.

\section{NOMENCLATURE}

$\begin{array}{ll}C_{1} & \text { leaned height at blade hub } \\ C_{2} & \text { leaned height at blade tip } \\ C a & \text { axial chord } \\ C p & \text { static pressure coefficient } \\ L E & \text { Blade Leading Edge } \\ M a_{i s} & \text { isentropic Mach number } \\ M a_{R e} & \text { relative Mach number } \\ m_{P E} & \text { mass flow rate under near peak efficiency } \\ m_{S} & \text { condition } \\ N P E & \text { Neass flow rate under near stall condition } \\ & \end{array}$

$\begin{array}{ll}N S & \text { Near Stall } \\ P S & \text { Pressure Surface } \\ S M & \text { Stall Margin } \\ S S & \text { Suction Surface } \\ T E & \text { Blade Trailing Edge } \\ \alpha_{1} & \text { leaned angle at blade hub } \\ \alpha_{2} & \text { leaned angle at blade tip } \\ \pi_{P E}^{*} & \text { total pressure ratio under near peak } \\ \pi_{S}^{*} & \text { efficiency condition } \\ & \text { total pressure ratio under near stall } \\ & \text { condition }\end{array}$




\section{INTRODUCTION}

The usage of blade lean (i.e., leaned and curved blades) has significant effect on the internal flow structures in axial compressors. Proposed by Deich et al. (1962) in the 1960s, blade lean has been extensively studied to better reveal the control mechanism on blade loading distribution, corner separations, etc (Welngold et al. 1995, 1997).

Sasaki and Breugelmans (1998) proposed a definition of blade lean and found that the intersection angle between blade suction surface and endwall should be higher than $90^{\circ}$ so as to improve the flow field. Weingold et al. (1995, 1997) developed a novel leaned compressor stator blade, which was equipped with blade lean at the end of blade so as to suppress the corner separation; in addition, blade lean was combined with controlled diffusion airfoil technology in their study. Results showed that $20 \%$ loss reduction was observed when the leaned angle of blade was set to $15^{\circ}$; positively leaned blade exhibited superior performance than that of negatively leaned blade; for the positively leaned blade, the loading in the adjacent region of blade end was reduced, whereas that at mid-span was increased. Breugelmans (1985) also found that leaned angle and leaned height of blade had considerable influence on the control effect of flow field. In the experimental study of Benini and Biollo (2007), blade lean was performed in a transonic compressor rotor, which resulted in alteration of shock wave structure and improved aerodynamic performance. Wang et al. (1981) investigated positively leaned blade in a compressor cascade, and the results showed that the radial secondary flow on suction surface was increased compared with linear blade; in addition, the cross-passage pressure gradient was reduced, resulting in reduced secondary flow. It was pointed out that the key flow mechanism was the redistribution of spanwise and chordwise loading.

Due to the significant effect of three-dimensional corner separation on compressor performance (Liu et al. 2020; Sun et al. 2021; Tang et al. 2020), various studies have been carried out on blade lean. The influence of strongly leaned stator blade was investigated based on a four-stage axial compressor developed Hannover University (Fischer et al. 2004). It was shown that the increased fraction due to the strongly leaned stator resulted in losses of static pressure ratio and efficiency at design condition and choking condition. Takahashi et al. (2005) compared the flow structure and performance of linear and leaned compressor cascades. Results showed that the leaned cascade reduced the vortex in the suction corner due to decreased endwall loading as compared with liner compressor. Kan et al. (2020) elucidated the effect of blade lean on a linear compressor cascade, and found that blade lean reduced the total flow loss by weakening the endwall vortex structure. Cao et al. (2017) studied the coupled influence of blade lean and boundary layer suction on the flow physics of a highly loaded compressor cascade, and the results indicated that blade lean effectively removed the corner separation and redistributed spanwise blade loading. Cao et al. (2019) comparatively analyzed the influence mechanisms of blade lean and non-axisymmetric endwall profiling on the control of corner separation and found that the influence of lean was on the whole blade span whereas the impact of non-axisymmetric end-wall profiling was on the local flow field near the endwall.

Although blade lean has been extensively studied in transonic compressor, the influence mechanism still needs to be revealed further because of the complex flow field which is featured by the interaction of shock wave with boundary layer and tip leakage flows. Oyama et al. (2003) from NASA Glenn Research Center carried out 3D lean and sweep distributions optimization design for the NASA 67 rotor blade. The optimization was carried out under the constraint of inlet mass flow rate and total pressure ratio. Results showed that the entropy production was reduced by more than $16 \%$, and the optimized design was featured with lambda-shaped shock wave on the suction side. The effect of blade lean on the stall margin was not considered in the study. Razavi and Boroomand (2014) investigated the influence of tangential blade lean and sweep on the operating parameters of the NASA rotor 67 and the results showed that leaned and swept blade had higher efficiency, total pressure ratio and stall margin than baseline rotor. Biollo and Benini (2009) found that a newly designed 3D swept and leaned rotor showed a higher efficiency which was due to a 3D modification of the shock wave structure. Sun et al. $(2019,2020)$ illustrated that the blade sweep had significant improvement effect on stall margin of a new-type low-reaction ultra-high-load transonic compressor rotor, which was due to the downstream migration of shock and the reduction of separation bubble on suction surface.

Tip leakage flow (Perrin and Leboeuf 1992; Shi and Fu 2013; Qiao et al. 2019) and its interaction with passage shock wave (Chunill and Rabe Douglas 2004) are the main flow structures near the casing of transonic compressor rotors, which are related to loss production and stall inception (Sun et al. 2020). Yamada et al. (2004) revealed the flow phenomena of tip leakage vortex in a transonic compressor rotor. In their study, a spiraltype breakdown of tip leakage vortex occurred due to the interaction with shock wave, which lead to a large blockage and self-sustained flow oscillation near the casing. Li et al. (2018) investigated the effect of chordwise nonuniform tip clearance on the performance of a transonic axial compressor. Results showed that nonuniform tip clearance effectively reduced the leakage mass flow rate, leading to enhancement of overall performance of the transonic compressor. An et al. (2018) analyzed the blade tip unsteadiness in a transonic compressor using NASA Rotor 35 . The unsteady results indicated that the shock/tip leakage vortex interaction generated the tip leakage vortex breakdown and caused a U-type vortex, whose periodic migration along blade pressure surface led to blade loading variation and flow unsteadiness near blade tip region; the shock oscillation was 
observed as indication of flow unsteadiness. Ahmad et al. (2020) numerically investigated the shock/tip leakage vortex interaction and the performance enhancement based on NASA Rotor 37. Result showed that shock/tip leakage vortex interaction generated a stagnation zone near blade tip region, which caused flow instability and triggered the stall. Yan et al. (2021) analyzed the main cause of axial transonic compressor stall and found that the tip leakage vortex breakdown generated irreversible high flow loss region; tip leakage vortex breakdown migrated toward the middle of blade passage, causing near tip leading edge blockage and triggering the rotor stall. The shock wave/tip leakage flow interaction led to a sudden strong flow deceleration and a strong change in the vorticity distribution of the vortex, and vortex breakdown occurred depending on the intensity of the shock wave/tip leakage flow interaction (Hofmann and Ballmann 2002). In the study of Biollo and Benini (2009), it was found that 3D leaned and swept rotor introduced a blade-toblade shock located more downstream than in the radial stacked rotor blade, and a less detrimental shock/tip leakage flow interaction at low-flow coefficient was obtained. Chunill and Rabe Douglas (2004) investigated the interaction of tip leakage vortices and passage shock in a swept transonic compressor rotor. Results showed that a low momentum area occurred downstream of the passage shock, which initiated stall inception; in addition, the tip leakage vortex oscillated due to shock oscillation. Du et al. (2013) carried out an investigation of the influence of blade lean on the leakage flow of a stator hub clearance. Results showed that, the onset location of leakage flow moved forward after introduction of leaned blade, however, the strength of hub leakage flow was reduced due to the reduced maximum loading near blade end.

Based on the forgoing analyses, the influence mechanism of blade lean was analyzed in a transonic compressor rotor-NASA rotor 37 (Suder 1996) numerically. The stacking line of the rotor blade was redesigned, and the influence mechanism of blade lean on the performance and shock wave/tip leakage flow interaction was revealed. The influence of leaned angle and leaned height were studied, with emphasis put on the comparison of positively and negatively leaned effect, which gave rise to some new findings.

\section{GEOMETRIC PARAMETERS AND OPERATING CONDITIONS OF INVESTIGATED COMPRESSOR ROTOR}

The NASA rotor 37 , of which blade was radially stacked originally, was designed and tested at NASA Lewis Research Center (Suder 1996). After that, the rotor was investigated widely by researchers (Hofmann and Ballmann 2002; Li et al. 2018; Yamada et al. 2004). The representative geometric and aerodynamic parameters of transonic rotor 37 are shown in Table 1, and more detailed data can be referred to Ref. (Suder 1996).
Table 1. Geometric and aerodynamic parameters of transonic rotor 37

\begin{tabular}{|c|c|}
\hline Parameter & Value \\
\hline Number of blades & 36 \\
\hline Rotor inlet hub-to-tip ratio & 0.7 \\
\hline Aspect ratio & 1.19 \\
\hline Tip Solidity & 1.288 \\
\hline Design rotating speed, rpm & 17188 \\
\hline Tip speed, $\mathrm{m} / \mathrm{s}$ & 454.136 \\
\hline Tip clearance, $\mathrm{mm}$ & 0.356 \\
\hline Design total pressure ratio & 2.106 \\
\hline Design adiabatic efficiency & 0.877 \\
\hline Choking mass flow rate, $\mathrm{kg} / \mathrm{s}$ & 20.93 \\
\hline
\end{tabular}

\section{COMPUTIONAL FLUID DYNAMIC METHOD AND VALIDATIONS}

\subsection{Computational Fluid Dynamic Method}

In this study, a fully three-dimensional ReynoldsAveraged Navier-Stokes solver was utilized for steady-state numerical simulation. The turbulence model utilized was Spalart-Allmaras. Calculations were carried out by fixing the total pressure, total temperature, flow angle at the inlet boundary whereas varying the static pressure at the outlet boundary. In addition, all the walls were considered slip-free and adiabatic.

Numerical simulations were carried out within a single blade passage under periodic flow condition. Structured mesh was generated by AUTOGRID in NUMECA FINE/TURBO software. The "O-type" mesh was generated around the blade surface and inside the tip clearance to improve mesh quality. Figure 1 shows the mesh on the blade and endwall surface with the enlarged view near blade LE, TE and tip clearance.

The size and density of mesh near the blade and endwall surfaces of the transonic rotor were refined during simulation procedure so as to capture the boundary layer development accurately. The y+ contours on blade and endwall surface are shown in Fig. 2. The $y+$ is approximately 1.0 , which meets the requirements of the turbulence model selected.

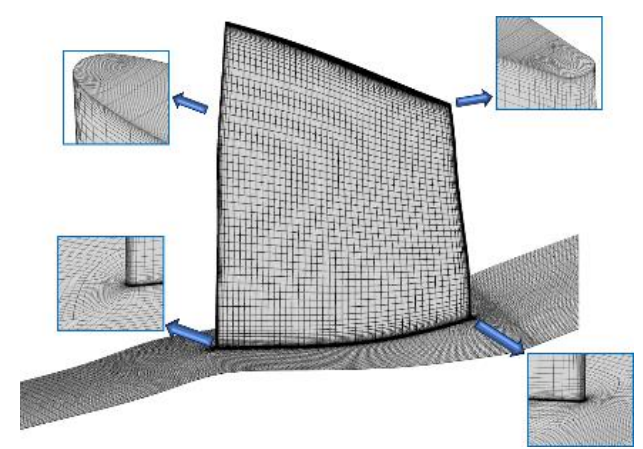

Fig. 1. Blade and endwall surface mesh of NASA rotor 37. 


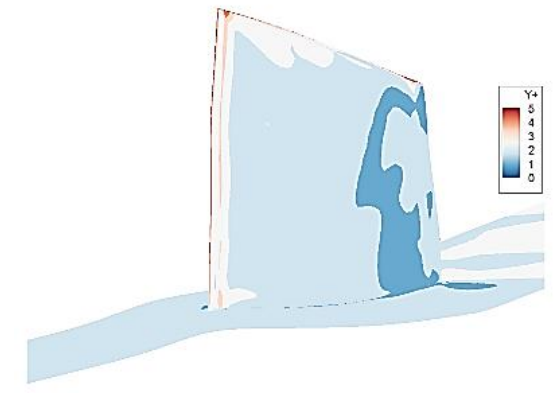

Fig. 2. $\mathrm{y}^{+}$contours near blade and endwall surfaces of baseline NASA rotor 37 .

\subsection{Validations of Computation Fluid Dynamic Method}

The mesh independence of the compressor was proved, based on which appropriate mesh density can be selected. Calculations were carried out at the design rotating speed. The lower mass flow rate operating point, which was the last converged steady computational condition, was obtained by increasing the outlet pressure with a small step (100 pa). The constant rotating speed performance lines of predicted results with number of nodes being approximately 0.5 to 4.0 million are shown in Fig. 3, where the constant rotating speed performance lines are expressed in the form of total pressure ratio and efficiency against normalized mass flow rate. The mass flow rates were normalized with the choking mass flow. It was demonstrated that as the number of nodes exceeded 2.0 million, the constant rotating speed performance lines did not change. As a consequence, the total number of nodes was approximately 2.0 million, and the number of radial nodes points inside the tip clearance was 37 so as to accurately solve to the tip clearance flow. The constant rotating speed performance lines of both predicted results and measured results are shown in Fig. 4. The predicted choking mass flow rate was $20.63 \mathrm{~kg} / \mathrm{s}$, whereas the measured value was $20.93 \pm$ $0.14 \mathrm{~kg} / \mathrm{s}$, indicating a small error between predicted result and measured result.

It was found that the constant rotating speed line was well captured with the computational method, although some points were out of the experiment uncertainties. Disagreements were observed in efficiency performance curve, where the predicted efficiency was $3.91 \%$ lower than that of measured results. The discrepancies of efficiency can be found in many exiting literatures on NASA rotor 37 , some of which have been shown in Fig. 4 for comparison (Ameri 2009; Sun et al. 2020). Disagreements were also found at the low-mass flow operating conditions, and mass flow rate obtained by calculation was lower than that of obtained by experiment. A number of researchers have achieved lower mass flow rate under NS operating condition of NASA rotor 37, such as Ameri (2009) and Sun et al. (2020). The reason is not well understood, but it is worth noting that the performance of compressor NS point, where there are significant flow unsteadiness and flow separation inside the blade passage, is difficult to be captured by either steady numerical method or experimental method.

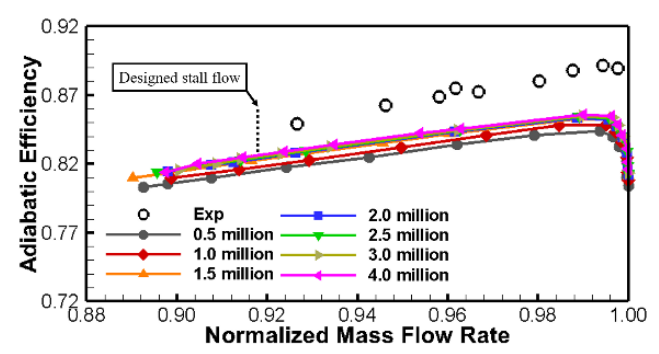

(a) Efficiency

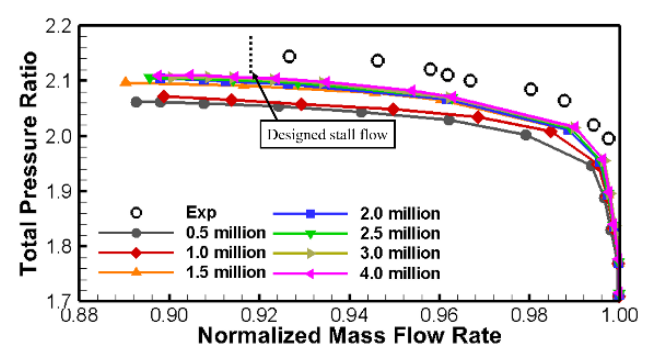

(b) Total pressure ratio

Fig. 3. Grids independence validation.

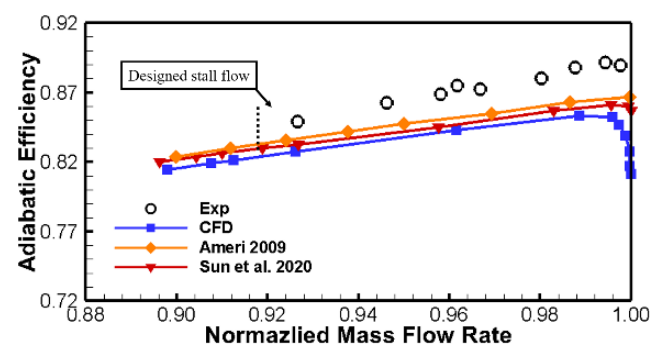

(a) Efficiency

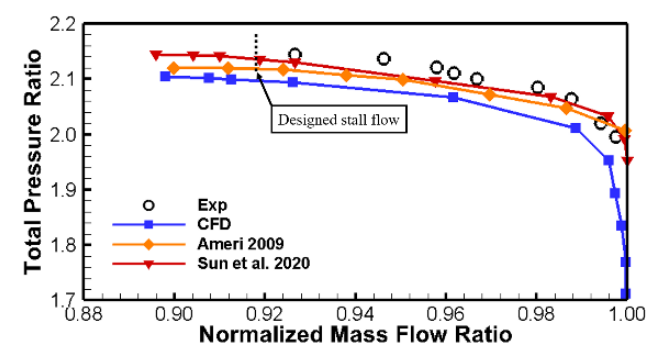

(b) Total pressure ratio

Fig. 4. Comparison of predicted and measured constant design speed lines of NASA rotor 37.

The calculated and measured circumferentiallymass-averaged spanwise performances (as shown in Fig. 5) indicate that the flow field was well predicted. The calculated results of total pressure ratio and efficiency distributions at $98 \%$ and $93 \%$ choking mass flows were compared with measured results. Despite some discrepancies shown at the outer span regions for both total pressure ratio and efficiency distribution, the shape of profiles was well captured. 


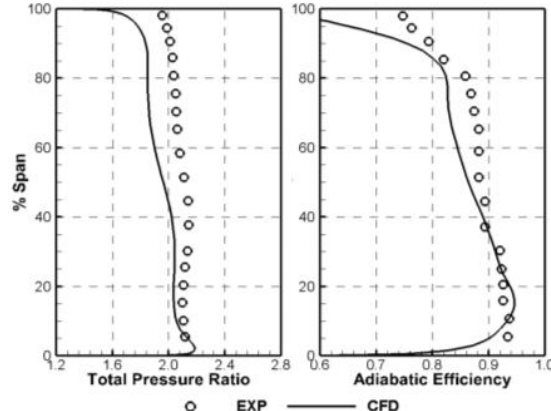

(a) $98 \%$ choking mass flow

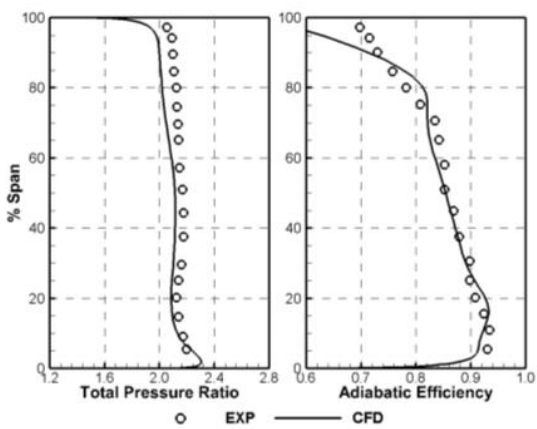

(b) $93 \%$ choking mass flow

Fig. 5. Comparison of predicted and measured spanwise distribution of NASA rotor 37.
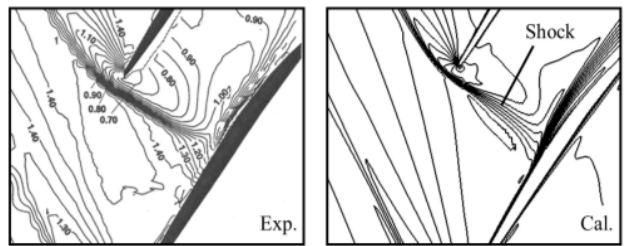

Fig. 6. Comparison of predicted and measured Mach number contours at $70 \%$ span.
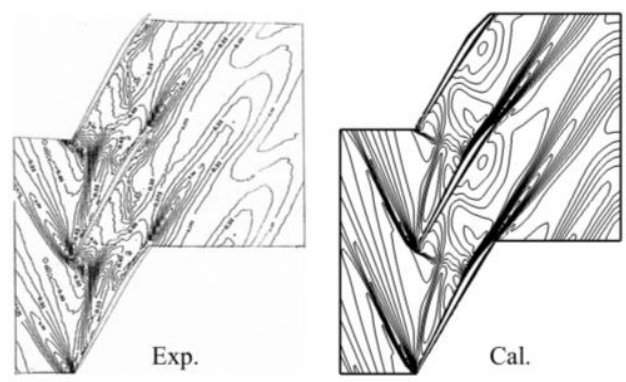

Fig. 7. Comparison of predicted and measured Mach number contours at $\mathbf{9 8 \%}$ span (shock wave/tip leakage flow interaction).

The comparison of blade-to-blade view, shock wave structure at $70 \%$ blade span and shock wave/tip leakage flow interaction at $95 \%$ blade span shows pretty good accuracy of the computational method (Fig. 6 and Fig. 7). Although it is well known that predicting the flow structure of shock wave and tip leakage flow interaction under casing boundary layer is a difficult task with steady-state solver, the flow features at $95 \%$ blade span of the NASA rotor 37 was calculated with acceptable accuracy

\section{LEANED NASA ROTOR 37 BLADE GEOMETRY}

The baseline NASA rotor 37 was stacked radially through the center of gravity of each blade section. Newly designed leaned rotor blades were obtained based on the baseline data by changing the stacking line in the tangential plane (the stacking line in the meridional plane remain unchanged), as shown in Fig. 8. The abscissa stands for the tangential direction, and the ordinate stands for spanwise direction. As shown in Fig. 8, the stacking line was defined with 6 control points. The 3 control points at outer span and the other 3 at inner span form two Bezier curves, which control the leaned stacking line shape near blade tip and hub region, respectively. In the middle blade span, the stacking line is kept linearly distributed. The leaned angle is defined as the angle between radial stacking line and the modified stacking line at blade hub $(\alpha 1)$ and blade tip $(\alpha 2)$. In the leaned blade cases of this study, $\alpha 1$ is equal to $\alpha 2$. Positively leaned blade is defined so that the pressure surface is pointing toward the endwall. Thus, the stacking line shown in Fig. 8 is negatively leaned blade. The height of leaned stacking line, i.e., $\mathrm{c} 1$ and $\mathrm{c} 2$ in the figure (in this study, $\mathrm{c} 1=\mathrm{c} 2$ ), is defined as leaned height. In order to reveal the influence of leaned angle effect and leaned height effect on the performance and shock wave/tip leakage flow interaction, 6 positively leaned rotor blades and 6 negatively leaned rotor blade were designed and numerically investigated and the samples of leaned transonic compressor rotor blades are shown in Fig. 9.

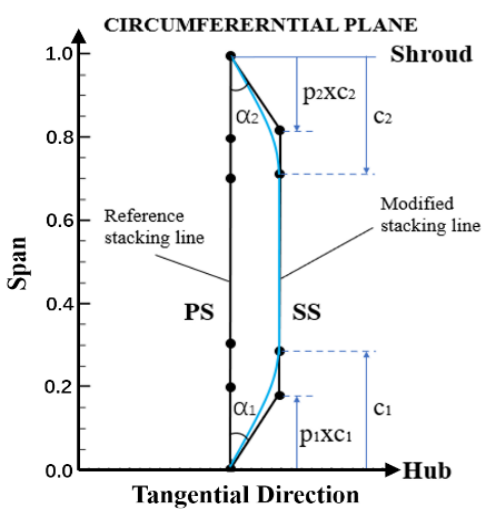

Fig. 8. Design of leaned blade by revising stacking line.

\section{RESULTS AND DISCUSSIONS}

\subsection{Influence of Positively Lean on Overall Performance of Transonic Rotor}

The constant speed lines for baseline transonic rotor and positively leaned rotor are shown in Fig. 10 and Fig. 11, respectively. The mass flow rates were 
normalized with choking mass flow rate of baseline rotor. It can be seen that both the efficiency and total pressure ratio of the whole constant speed line (except the choking condition) of positively leaned rotor were decreased compared with baseline rotor. However, exceptions were observed when the leaned height was 0.3 and leaned angle was $5^{\circ}$, which exhibited slightly higher total pressure ratio

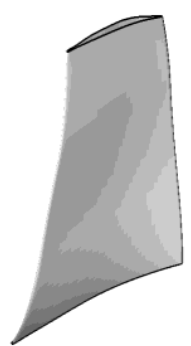

(a) positively leaned blade

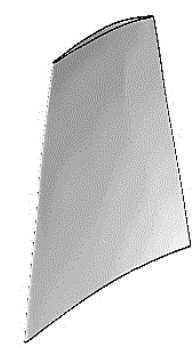

(b) baseline blade (c)

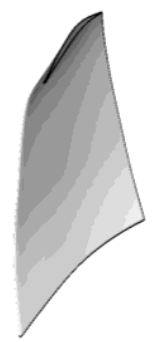
negatively leaned blade
Fig. 9. Samples of leaned transonic compressor rotor blades.

in some operating conditions. It was interesting to see that positively leaned blade improved the endwall flow field of compressor (it means higher performance), due to the reduction of loading near blade end. The findings are opposite with the conventional view.

As leaned angle increased from $0^{\circ}$ (baseline rotor) to $20^{\circ}$, it was observed (in Fig. 10) that both efficiency and total pressure ratio were decreased. At leaned angle of $20^{\circ}$ (the highest leaned angle in this study), the absolute value of peak efficiency was reduced by as much as 4.34 , the maximum reduction of peak total pressure ratio was 0.1 . It was worth noting that the total pressure ratio of $5^{\circ}$ leaned rotor increased slightly compared with that of baseline configuration. Despite the efficiency and total pressure ratio reduction under positively leaned angle, the mass flow rates at stall limit of $5^{\circ}$ and $10^{\circ}$ leaned angle were reduced obviously compared with that of baseline configuration, and the choking mass flow rates of all 4 cases were increased, indicating that the stall margin was increased under low leaned angles. The stall margins of all positively leaned rotors are shown in Fig. 12. The stall margin is defined in Equation 1 as follows,

$S M=\left(\frac{\pi_{S}^{*} / m_{S}}{\pi_{P E}^{*} / m_{P E}}-1\right) \times 100 \%$

where $\pi_{S}^{*}$ and $\pi_{P E}^{*}$ are the total pressure ratio under near stall (NS) and near peak efficiency (NPE) condition, respectively; $m_{S}$ and $m_{P E}$ are mass flow rate under NS and NPE condition, respectively. The case of $5^{\circ}$ leaned angle featured the maximum stall margin, which was $8.36 \%$ higher in absolute value and $54.97 \%$ higher in relative value than that of baseline configuration.
Under identical leaned angle of $20^{\circ}$, as shown in Fig. 11 , it can be seen that both efficiency and total pressure ratio were decreased as leaned height increased from 0.1 to 0.3 (span). The peak efficiency was reduced by $4.34 \%$ at most under leaned height of 0.3. The maximum total pressure ratio was decreased by 0.1 at most. Being similar to the variations with leaned angle, it's shown that the mass flow rate at stall limit under low leaned height $(0.1)$ was reduced, and the choking mass flow rates of all leaned height were increased, indicating that the stall margin was increased under low leaned height. The stall margin was increased by $6.23 \%$ at most in the figure. However, as leaned height increased, the stall margin reduced remarkably due to the significant attenuation of rotor performance.

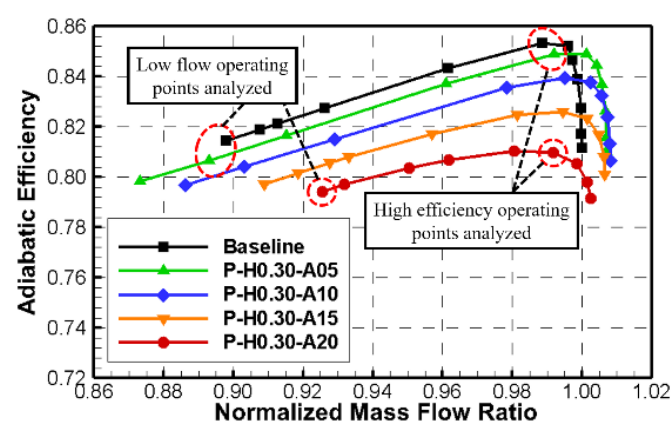

(a) Efficiency performance

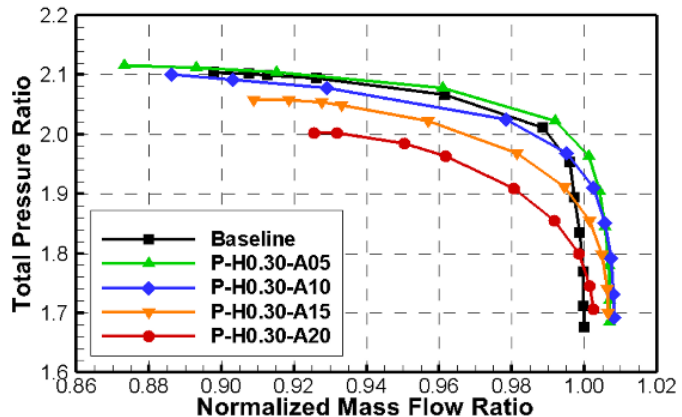

(b) Total pressure ratio performance

Fig. 10. Comparison of constant speed line under identical leaned height.

\subsection{Influence of Positively Lean on Spanwise Performance and Flow Structure of Transonic Rotor}

In order to reveal the flow mechanism of blade lean on the transonic compressor rotor, the results of two leaned cases, i.e., P-H0.30-A05 and P-H0.30-A20, are presented for further analysis. The case P-H0.30A05 exhibited optimum predicted efficiency, total pressure ratio and stall margin among the all positively leaned cases, whereas P-H0.30-A20 showed the lowest predicted performance. The spanwise pitchwise mass averaged aerodynamic parameters (acquired from 70\% axial chord downstream of blade TE) and flow fields are presented in the following. 


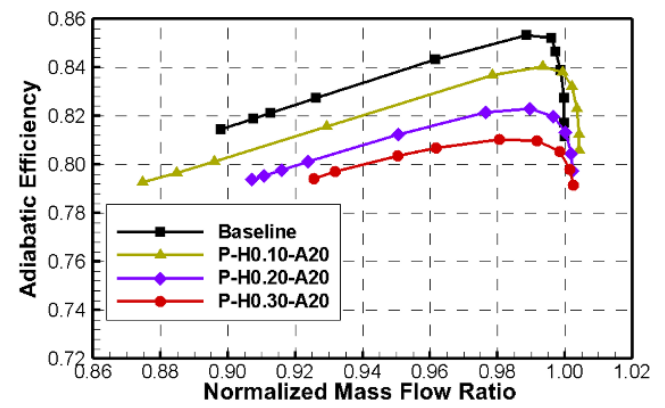

(a) Efficiency performance

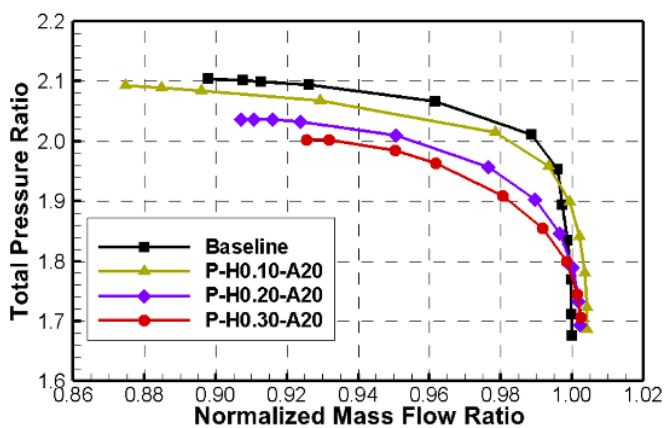

(b) Total pressure ratio performance

Fig. 11. Comparison of constant speed line under identical leaned angle.

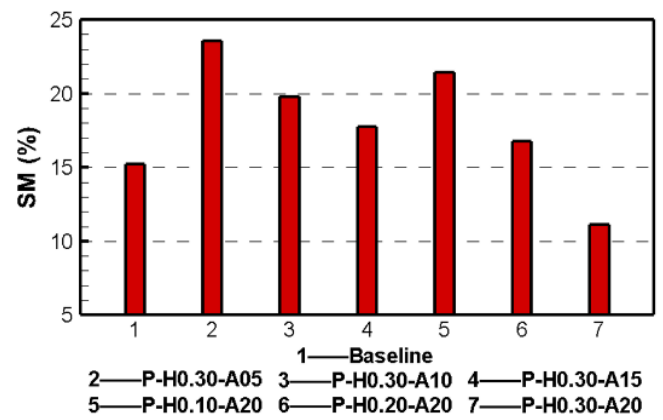

Fig. 12. Comparison of stall margin of all positively leaned rotors.

Under NPE condition, as shown in Fig. 13, the spanwise efficiency distribution of leaned rotor PH0.30-A05 was approximately the same as that of baseline configuration, except the region between $50 \%$ and $95 \%$ spans where slight decrement of efficiency was observed. As leaned angle increased to $20^{\circ}$, the efficiency of the whole span was decreased, and the maximum decrement was observed at $50 \%$ to $100 \%$ spans, which was consistent with that of P-H0.30-A05 case. The efficiency decrement was due to the interaction of shock/boundary layer. Compared with baseline configuration, it was interesting to find that the total pressure ratio distribution was reduced along the entire blade span as positively leaned angle increased.

Figure 14 shows the spanwise performance profiles under low mass flow (NS) condition. As leaned angle increased, within the region from $70 \%$ to $100 \%$ span and from 0 to $30 \%$ span, the efficiency reduced gradually. The total pressure ratio of rotor P-H0.30A20 exhibited considerable decrement compared with baseline rotor along the entire span.

Numerical results showed a considerable variation of shock structure in the positively leaned rotor passage, which led to the change of efficiency and total pressure ratio. Under NPE condition, at $75 \%$ blade span, the bow shock and passage shock of baseline rotor were coalesced into a single one (Fig. 15 (a)) at the entrance of the blade passage. With the increase of leaned angle, the double-shock structure emerged, i.e., bow shock and passage shock separated from each other. The peak value of relatively Mach number near suction surface increased considerably (Fig. 15(b)), which resulted in increased strength of shock-boundary layer intersection. The width of wake at $75 \%$ span was enlarged obviously. The shock-induced separation led to the reduction of efficiency and flow turning (total pressure ratio). As consequence, the flow diffusion was decreased for P-h0.30-A20 case, which was consistent with the results in Fig. 13 and Fig. 14.

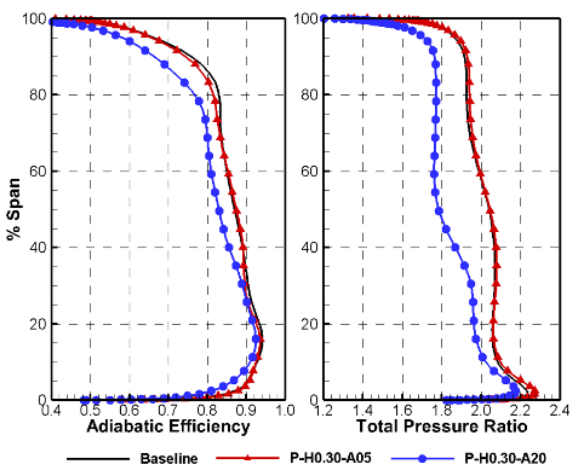

Fig. 13. Spanwise performance profiles at NPE condition.
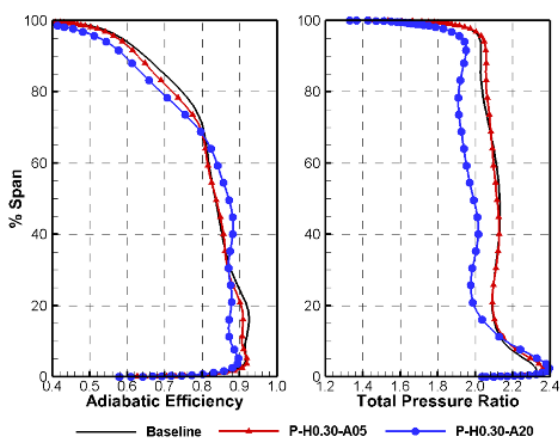

Fig. 14. Spanwise performance profiles at NS condition.

Figure 16 shows the comparison of flow field associate with the shock location, flow separation and static pressure on blade suction surface. At mid span, it can be seen that the impinging location of 

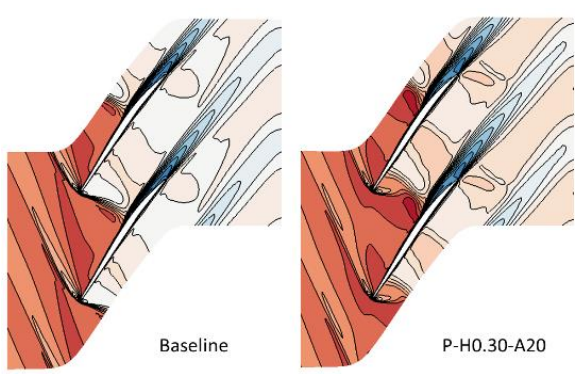

$\begin{array}{lllllllllllllllll}M a_{\mathrm{pe}}: & 0.1 & 0.2 & 0.3 & 0.4 & 0.5 & 0.6 & 0.7 & 0.8 & 0.9 & 1 & 1.1 & 1.2 & 1.3 & 1.4 & 1.5 & 1.6\end{array}$

(a) Relative Mach number contours

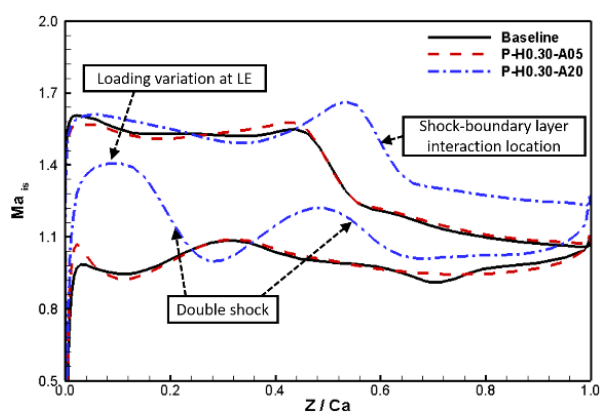

(b) Isentropic Mach number distribution on blade surface

Fig. 15. Comparison of flow field at $75 \%$ blade span for baseline rotor and leaned rotor, NPE condition.
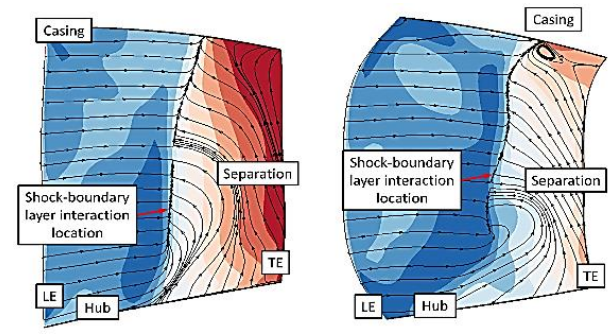

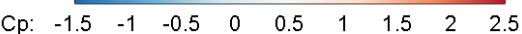

(a) Baseline rotor

(b) $\mathrm{P}-\mathrm{H} 0.30-\mathrm{A} 20$ rotor

Fig. 16. Comparison of near suction surface flow field, NPE condition.

shock for positively leaned rotor on blade suction surface shifted downstream; in addition, the static pressure was reduced at upstream of shock impinging location, which indicates that the shock front Mach number was increased. Although the flow separation region was reduced, the loss is increased and the diffusion is reduced due to the stronger shock-boundary layer interaction. Near the hub, the single shock of the baseline rotor was replaced with a double-shock structure for the leaned rotor. The shock induced boundary layer separation line is disappeared, the flow separation also occurs. Thus, the efficiency near the hub was approximately the same as that of baseline rotor. Near blade tip, it was observed that the shock location shifted toward trailing edge because of the viewing angle (which will be explained in the following, Fig. 17); actually, the shock location shifted upstream in positively leaned rotor.

\subsection{Influence of Positively Lean on Shock Wave/Tip Leakage Flow Interaction}

Figure 17 shows the relative Mach number contours at blade tip span location (98\% span) and 3D tip leakage flow streamlines under NPE condition. The streamlines were released at mid-height in the tip clearance gap, from leading edge to trailing edge. The dashed line in yellow stands for the tip leakage vortex core (or primary vortex).

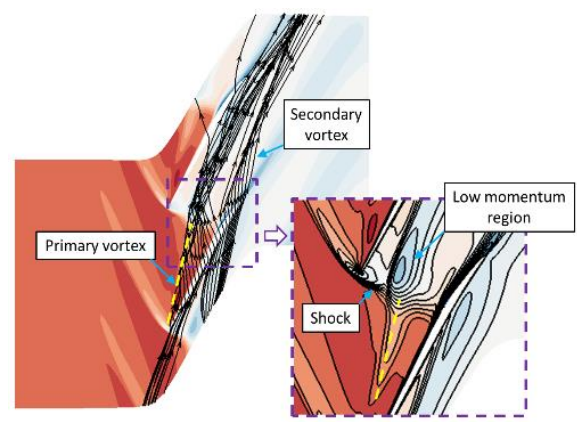

(a) Baseline rotor

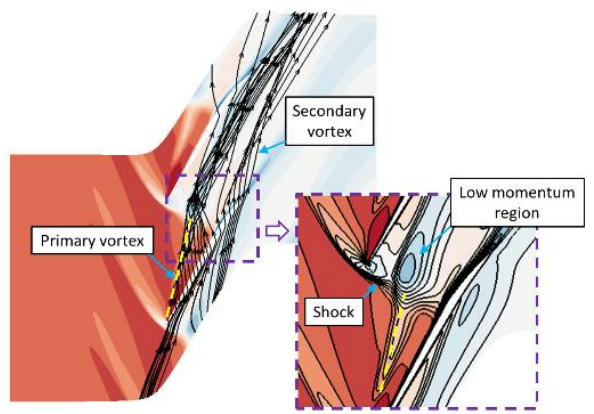

(b) $\mathrm{P}-\mathrm{H} 0.30-\mathrm{A} 05$ rotor

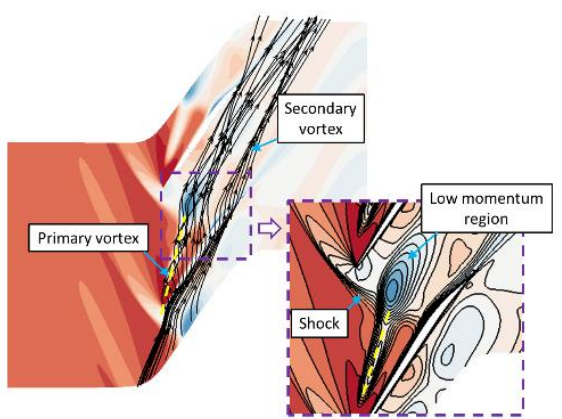

(c) P-H0.30-A20 rotor

$\begin{array}{lllllllllllllllll}\mathrm{Ma}_{\mathrm{Rg}}: & 0.1 & 0.2 & 0.3 & 0.4 & 0.5 & 0.6 & 0.7 & 0.8 & 0.9 & 1 & 1.1 & 1.2 & 1.3 & 1.4 & 1.5 & 1.6\end{array}$

Fig. 17. Blade-to-blade relative Mach number contours \& tip leakage flow streamlines, $98 \%$ span, NPE condition.

For baseline case, the vortex originated at blade leading edge; after the shock, the primary vortex was enlarged and low-momentum region appeared due to 
shock/tip leakage vortex interaction. From 3D streamlines, it is shown that the tip leakage vortex (primary vortex) was clearly visible. The 3D streamlines can be divided into two parts, i.e., one is the primary vortex and the other is secondary vortex which arises after the shock. The primary vortex arises from the pressure difference between the pressure side and suction side before the shock, whereas the secondary vortex arises from suction side separated fluid and the primary vortex fluid (which flows through the blade tip for the second time). As leaned angle increased, the shock moved upstream and the shock/tip leakage vortex interaction point shifted upstream, which was due to the increase of loading near the leading edge of blade tip (Fig. 17). The starting point of primary vortex was also located at blade leading edge, and the trajectory of primary vortex was more inclined to pitchwise. As a result, the low-momentum region after the shock increased and its minimum Mach number value reduced considerably as leaned angle increased. Moreover, the secondary vortex decreased with the increase of leaned angle, which was due to the reduction of loading after the shock (Fig. 17).

Figure 18 shows the blade loading (isentropic Mach number) distribution at $98 \%$ span under NPE condition. At low leaned angle, the loading distribution was approximately the same as that of baseline case, although the loading near leading edge was increased. For P-H0.30-A20 rotor, in the region between 0 and 0.32 axial chord, the loading was increased considerably compared with baseline rotor; from 0.32 to trailing edge, the loading was reduced significantly. As a whole, at high leaned angle, the loading at blade tip was increased and the static pressure at trailing edge was reduced.

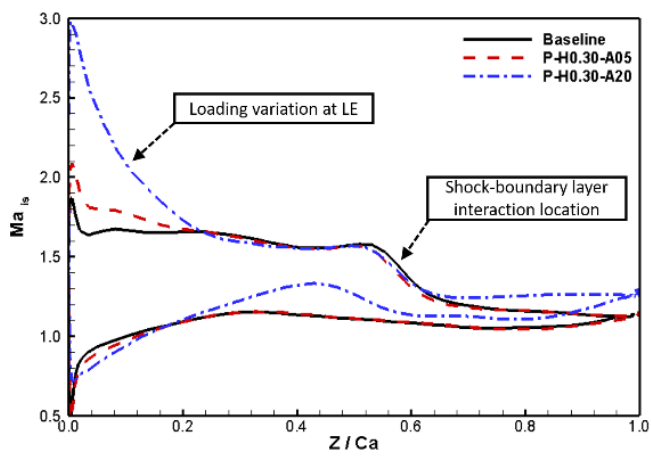

Fig. 18. Isentropic Mach number distribution on blade surface, $98 \%$ span, NPE condition.

It was interesting to see that the loading of positively leaned rotor was increased and the overall static pressure was decreased at blade tip, which is opposite to conventional view. The mechanism was that blade lean by pitchwise barreling blade sections led to blade backward swept in the 3D rotor, and the backward swept effect was more significant at the location where the settle angle was high (rotor tip section). Backward swept resulted in increased loading at fore part of tip region, and reduced loading at aft part, where the loading fell down to 0 (no blade).

Figure 19 shows the flow field associated with tip leakage vortex and shock under NS condition. The flow field can help to understand the mechanism of the variation of stability margin for positively leaned rotor. For baseline rotor, there were also primary vortex and secondary vortex near blade tip. Considering higher blade loading under NS condition, than NPE condition, the tip leakage was enlarged significantly and broken down after the shock; in addition, the relative Mach number was lower than that of NPE condition and the low relative Mach number area was larger, occupying most the area of blade passage at blade tip. Under low leaned angle (the case of P-H0.30-A05), the 3D tip leakage streamlines were similar as that of baseline rotor; the low relative Mach number region was slightly smaller than that of baseline rotor, which is consistent with the increase of stall margin (under numerical results). For high leaned angle, the phenomenon of vortex breakdown was enhanced, and the low relative Mach number region is enlarged significantly compared with baseline rotor, which is also consistent with the variation of stall margin.

\subsection{Influence of Negatively Lean on Performance and Flow Structure of Transonic Rotor}

Figure 20 and Fig. 21 show the comparison of constant speed line for negatively leaned rotor and baseline rotor. The mass flow rates were normalized with the chocking mass flow of baseline rotor. It can be seen that the efficiency and total pressure ratio were increased at appropriate leaned height and leaned angle. The peak efficiency of N-H0.30-A05, $\mathrm{N}-\mathrm{H} 0.30-\mathrm{A} 10$ and $\mathrm{N}-\mathrm{H} 0.30-\mathrm{A} 15$ rotors was higher than that of baseline rotor; the total pressure ratio of $\mathrm{N}-\mathrm{H} 0.30-\mathrm{A} 05$ rotor is higher than that of baseline rotor.

As shown in Fig. 20, as negatively leaned angle increases from $0^{\circ}$ (baseline rotor) to $20^{\circ}$, the peak efficiency and total pressure ratio showed a tendency of first increasing and then decreasing compared with that of baseline case. At $5^{\circ}$ leaned angle, the peak efficiency was increased by $0.8 \%$ at most, and the maximum increment of total pressure ratio reached 0.05 . The choking mass flow rate decreased due to the blockage of flow separation at low blade span for negatively leaned rotors. As shown in Fig. 21 , as leaned height increased (the leaned angle was fixed at $20^{\circ}$ ), the peak efficiency and the total pressure ratio (on the whole constant speed line) reduced gradually, and the chocking mass flow rate decreased as well. For all negatively leaned cases, since there was no obvious variation of the mass flow rate difference between chocking and NS condition, the stall margin remained unchanged approximately. 


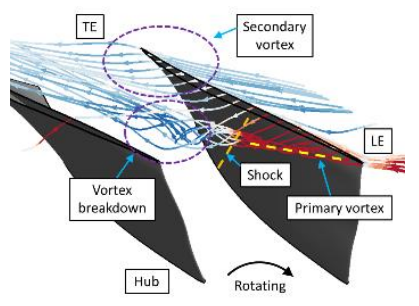

(a) Baseline rotor

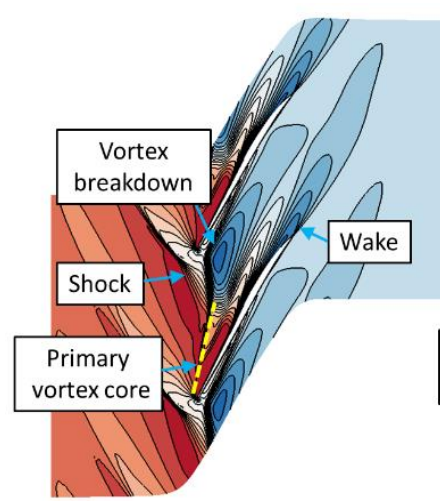

(d) Baseline rotor

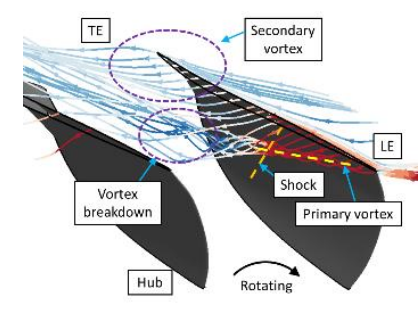

(b) $\mathrm{P}-\mathrm{H} 0.30-\mathrm{A} 05$ rotor

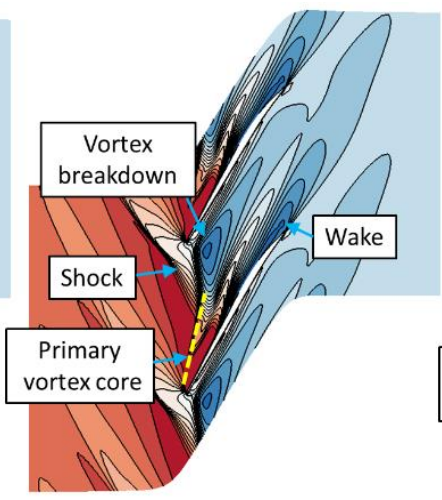

(e) P-H0.30-A05 rotor

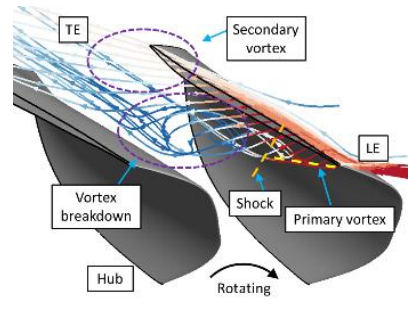

(c) P-H0.30-A20 rotor

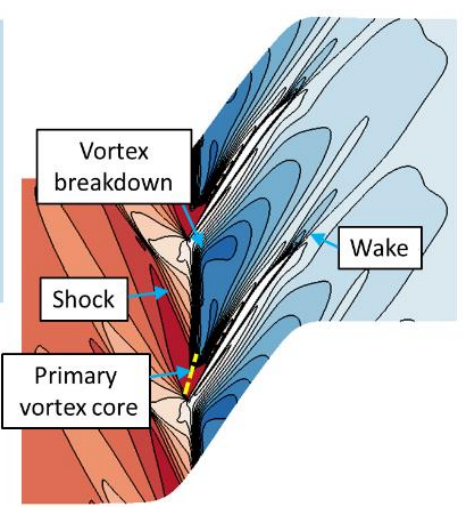

(f) P-H0.30-A20 rotor

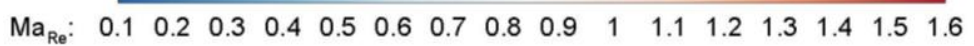

Fig. 19. Isentropic Mach number distribution on blade surface, $98 \%$ span, NS condition.

The stall margin of negatively leaned rotors is shown in Fig. 22. It is clear that the predicted stall margin of negatively leaned rotors exhibited no obvious variation compared with positively leaned rotor. For $\mathrm{N}-\mathrm{H} 0.30-\mathrm{A} 05$ rotor, although the mass flow rate region between choking and NS condition was reduced, the predicted stall margin increased slightly, which was because the stall margin was calculated with the value under NPE condition and NS condition (the mass flow rates were both reduced). For N-H0.30-A20, the stall margin was increased considerably (the mass flow range did not show significant variation), which was due to the reduction of mass flow rates and the increase of the ratio of total pressure ratio between NS condition and peak efficiency condition.

Two negatively leaned rotors, i.e., N-H0.30-A05 and $\mathrm{N}-\mathrm{H} 0.30-\mathrm{A} 20$, were selected to further study the flow mechanism. N-H0.30-A05 rotor exhibited maximum peak efficiency, whereas N-H0.30-A20 featured the maximum reduction of peak efficiency and total pressure ratio. The spanwise pitchwise mass averaged aerodynamic parameters (acquired from $70 \%$ axial chord downstream of blade TE) are shown in Fig. 23 and Fig. 24, respectively.

As shown in Fig. 23, the spanwise efficiency distribution between $50 \%$ and $95 \%$ spans of $\mathrm{N}$ H0.30-A05 rotor was higher than that of baseline rotor; the efficiency at other spans was approximately the same as that of baseline rotor. Under NS condition (as shown in Fig. 24), the

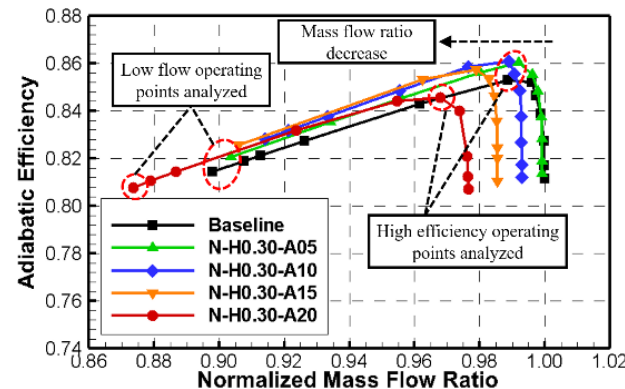

(a)

Efficiency performance

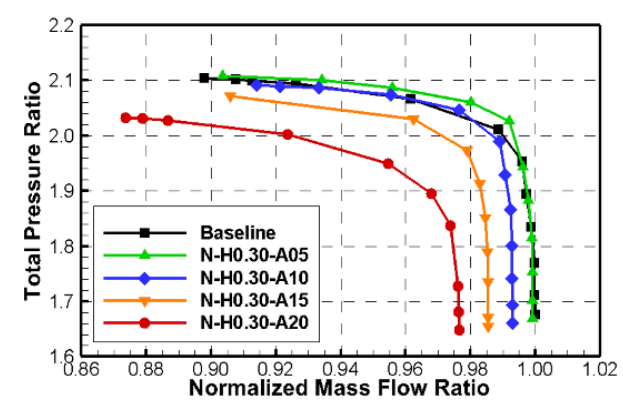

(b) Total pressure ratio performance

Fig. 20. Comparison of constant speed line under identical leaned height.

efficiency increment of N-H0.30-A05 rotor was also observed at $50 \%$ to $95 \%$ spans; the efficiency at other spans was approximately the same as that of baseline 


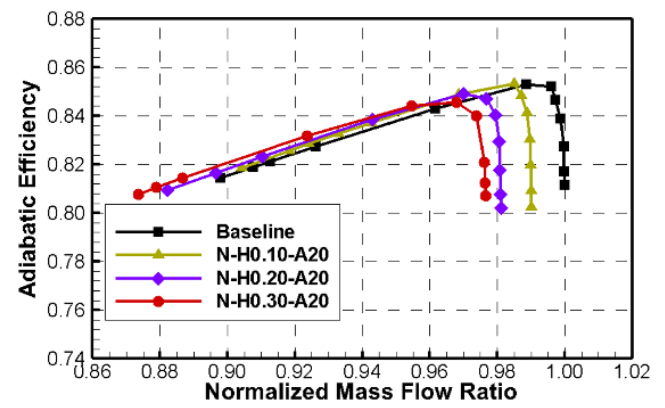

(a) Efficiency performance

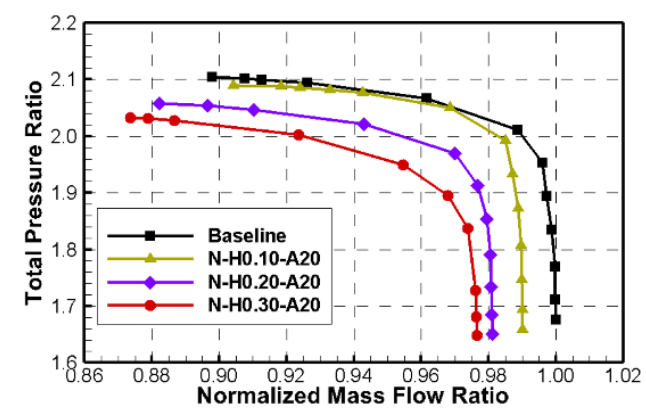

(b) Total pressure ratio performance

Fig. 21. Comparison of constant speed line under identical leaned angle.

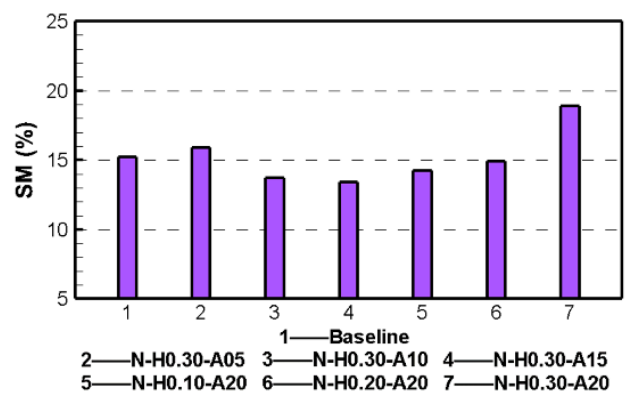

Fig. 22. Comparison of stall margin of all negatively leaned rotors.

rotor. In summary, the overall efficiency increment of constant speed line of N-H0.30-A05 rotor was observed at the region from $50 \%$ to $95 \%$ spans. Under both NPE and NS conditions, as negatively leaned angle increased to $20^{\circ}$, the efficiency in the region from $50 \%$ to $95 \%$ span increased considerably. Figure 25 shows that the shock induced boundary layer separation was suppressed significantly at negatively leaned angle of $20^{\circ}$, which was the flow mechanism of efficiency improvement. It was interesting to find that the efficiency of P$\mathrm{H} 0.30$-A20 rotor at 0 to roughly $50 \%$ span was reduced considerably, which was due to the flow separation caused by negatively leaned rotor.

Because of reduced shock/boundary layer interaction and increased efficiency, the total pressure increased near mid-span. Near blade tip, where the shock/boundary layer interaction was reduced, the total pressure was reduced rather than increased, which was due to the decrease of loading near blade tip (negatively leaned blade by pitchwise barreling blade sections resulted in forward sweep at high setting angle sections, i.e., blade tip section; forward sweep reduced the loading near blade tip region). The total pressure reduction near hub was caused by the flow separation.

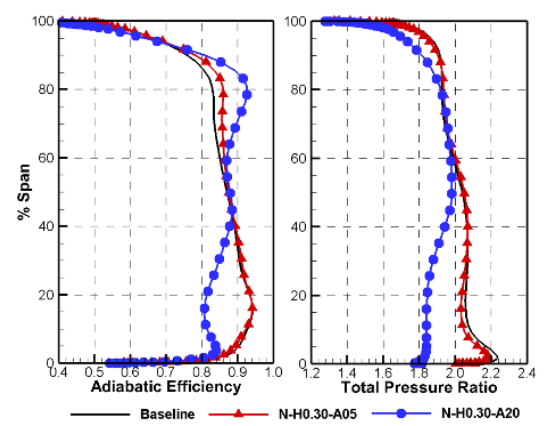

Fig. 23. Spanwise performance profiles at NPE condition.

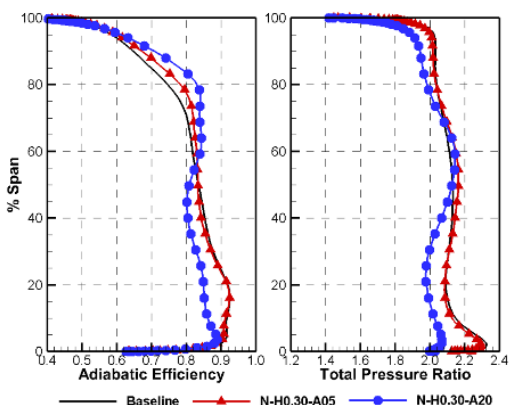

Fig. 24. Spanwise performance profiles at NS condition.

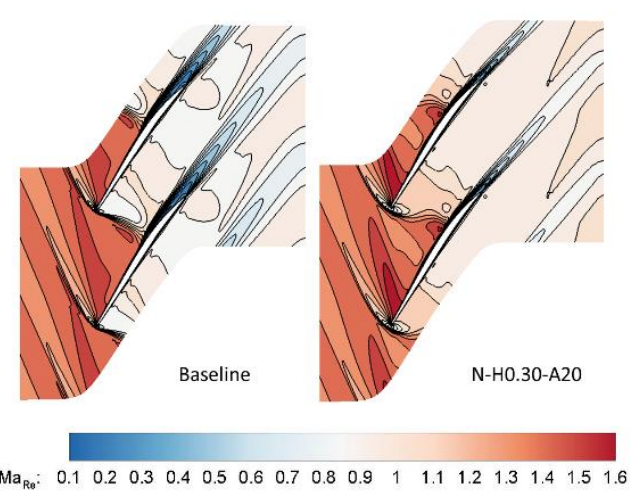

Fig. 25. Comparison of flow field at $75 \%$ blade span, NPE condition.

Numerical results of the negatively leaned rotors showed that the shock structure exhibited considerable variation, leading to the change of aerodynamic performance. Under NPE condition, at $75 \%$ blade span of $20^{\circ}$ negatively leaned rotor, the coalesced single shock structure tended to separate into a double-shock structure, i.e., bow shock and passage shock. However, the passage shock was not as obvious as that of positively leaned configuration. 
The peak relatively Mach number near the shock was increased compared with baseline rotor, however, the shock/boundary layer interaction did not increase (the shock-induced boundary layer separation was reduced significantly). The flow mechanism was that the 3D shock structure inside the rotor was inclined significantly (as shown in Fig. 26); it is widely acknowledged that the 3D inclined shock reduces the relatively Mach number normal to the shock front, leading to the decline of the shock/boundary layer interaction (Denton and $\mathrm{Xu}$ 2002).

Figure 26 shows the suction surface static pressure and limiting streamlines of baseline rotor and PH0.30-A20 rotor. It is clear that the shock foot of baseline rotor was roughly in a radial distribution; for negatively leaned rotor, the shock was inclined from hub to tip (the shock foot was located near trailing edge at blade tip). The 3D inclined shock resulted in higher efficiency at upper span, however, the static pressure and total pressure ratio were reduced. As shown in Fig. 26, negatively lean resulted in forward swept rotor, particularly for the tip region where the setting angle was the highest. As shown in Fig. 27, forward swept near tip caused reduced overall loading in the adjacent spanwise region; along chordwise, the loading from 0 to 0.5 axial chord of negatively leaned rotors was decreased significantly, whereas the loading of 0.5 to trailing edge is increased. With the increase of leaned angle, the shock front relative Mach number was increased considerably. Due to the inclined 3D shock structure, the increase of Mach number did not cause higher loss production. As the loading reduces, the static pressure at trailing edge was reduced, and the work input (total pressure ratio) was also decreased near blade tip (Fig. 23 and Fig. 24).

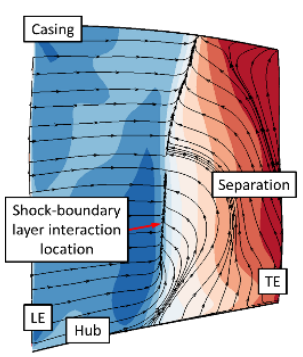

(a) Baseline rotor

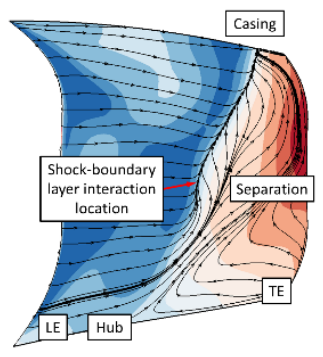

(b) N-H0.30-A20 rotor

$$
\text { Cp: } \begin{array}{lllllllll}
-1.5 & -1 & -0.5 & 0 & 0.5 & 1 & 1.5 & 2 & 2.5
\end{array}
$$

Fig. 26. Comparison of near suction surface flow field, NPE condition.

Figure 28 shows the blade-to-blade relative Mach number contours of $98 \%$ span and 3D streamlines released at mid-height of tip clearance of negatively leaned rotor under NPE condition, where the flow field of baseline rotor is also provided for comparison. The tip leakage vortex also contained primary vortex and secondary vortex. In the negatively leaned rotor, the primary vortex started at about 0.15 and 0.26 axial chord for $5^{\circ}$ and $20^{\circ}$

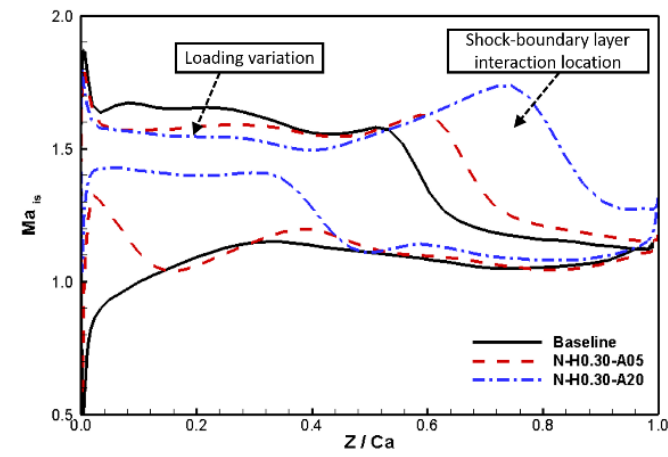

Fig. 27. Comparison of isentropic Mach number distribution on blade surface, $98 \%$ span, NPE condition.

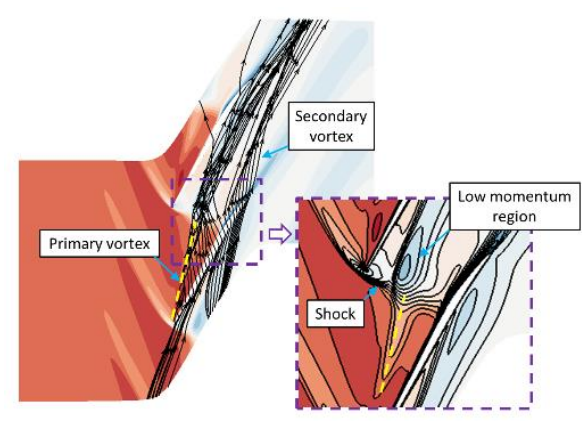

(a) Baseline rotor

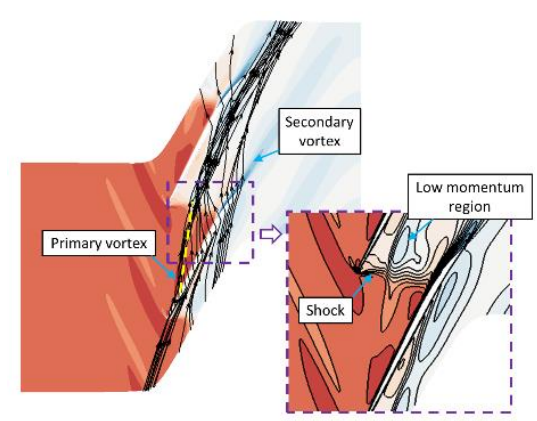

(b) $\mathrm{N}-\mathrm{H} 0.30-\mathrm{A} 05$ rotor

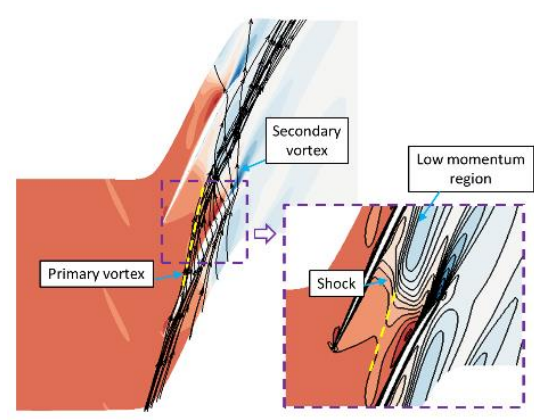

(c) N-H0.30-A20 rotor

$\begin{array}{lllllllllllllllll}\mathrm{Ma}_{\mathrm{Rg}}: & 0.1 & 0.2 & 0.3 & 0.4 & 0.5 & 0.6 & 0.7 & 0.8 & 0.9 & 1 & 1.1 & 1.2 & 1.3 & 1.4 & 1.5 & 1.6\end{array}$

Fig. 28. Blade-to-blade relative Mach number contours \& tip leakage flow streamlines, $\mathbf{9 8 \%}$ span, NPE condition. 
negatively leaned rotors, due to the lower blade loading near blade leading edge. Further, the shock/tip leakage vortex interaction location was located at more downstream for the negatively leaned rotors, which is the result of inclined 3D shock structures. However, the flow features do not have significant influence on the local efficiency distribution.

The shock front of leaned rotor was located more downstream compared with that of baseline configuration. In fact, it was no longer attached to the blade leading edge, but it became a shock inside the blade passage for the $20^{\circ}$ negatively leaned rotor. The variation of the shock front led to a larger distance between blade leading edge and shock front, which resulted in larger mass flow range between NS condition and peak efficiency condition. However, due to the deteriorated flow field at lower span of $20^{\circ}$ negatively leaned rotor, the trigger of stall became severe flow separation at lower span, so it did not increase the stall margin significantly. For N-H0.30A05 rotor, although the shock/tip leakage vortex interaction flow phenomenon was improved and the hub flow field was not deteriorated obviously, the stall margin was not enlarged in predicted results. This was probably due to the accuracy of numerical method on capturing NS condition at present. The difficulty in prediction of stall margin of the NASA rotor 37 was also found in the research of Ref. (Biollo and Benini 2009). The solution for this problem may be waiting for solving code with high accuracy or carrying out experimental research in the future.

\section{CONCLUSIONS AND PROSPECTS}

In a transonic compressor rotor, the influence mechanism of blade lean on performance and shock/tip leakage flow interaction was investigated by taking leaned pattern, leaned angle and leaned height as variables. Conclusions are drawn as follows:

(1) Compared with baseline rotor, the efficiency and total pressure ratio of the whole constant speed line of positively leaned rotor were decreased. At $20^{\circ}$ leaned angle, the absolute value of peak efficiency was decreased $4.34 \%$, the maximum reduction of peak total pressure reaching 0.01 . The backward sweep was introduced by pitchwise barreling leaned blade sections, generating high setting angle at blade tip, results in the opposite findings to the conventional view.

(2) Increasing positively leaned angle led to extensive shock/tip leakage vortex interaction and downstream migration of chordwise loading near mid-span. The single shock structure of baseline rotor separated into a double-shock structure, i.e., bow shock and passage shock. Higher shock front Mach number generate more extensive shock/boundary layer interaction, leading to thicker wake and lower efficiency in the adjacent region. Under NS condition, interacted by shock, tip leakage vortex breakdown occurs; the phenomenon will be enhanced as positively leaned angle increasing.
(3) For negatively leaned rotor, the origin of primary vortex moved downstream and the shock/tip leakage vortex interaction position also migrated toward downstream. As a consequence, an inclined 3D shock structure was generated. Due to the blockage of flow separation at low blade span, the choking mass flow rate reduced. The peak efficiency and total pressure ratio showed a tendency of first increasing and then decreasing with the increase of leaned angle. At leaned angle of $5^{\circ}$, the peak efficiency was increased by $0.8 \%$ at most, and the maximum increment of total pressure ratio reached 0.05 .

(4) For most positively leaned cases, the predicted stall margin increased, however, the shock front moved upstream and the shock/tip leakage vortex interaction was intensified. For negatively leaned rotors, the predicted stall margin did not show significant variation, however, the shock front moved downstream significantly and the shock/tip leakage vortex interaction was weakened. Such difference is perhaps due to the accuracy of numerical method on capturing NS condition at present. The solution may be waiting for high accuracy solving code or carrying out experimental research in the future.

\section{ACKNOWLEDGEMENTS}

This work was supported by National Natural Science Foundation of China (Nos. 51806174 and 51741601) and the Fundamental Research Funds for the Central Universities of China (No. G2018KY0303).

\section{REFERENCES}

Ameri, A. A. (2009). NASA Rotor 37 CFD Code Validation Glenn-HT Code. AIAA Aerospace Sciences Meeting Including the New Horizons Forum and Aerospace Exposition. AIAA 20091060.

An, G, Y. Wu and J. Lang (2018). Numerical Investigations into the Origin of Tip Unsteadiness in a Transonic Compressor. Journal of Applied Fluid Mechanics 11(4), 1133-1141.

Ahmad, N., J. Bin and Z. Qun (2020). Performance Enhancement of a Transonic Axial Flow Compressor with Circumferential Casing Grooves to Improve the Stall Margin. Journal of Applied Fluid Mechanics 13(1), 221-232.

Benini, E. and R. Biollo (2007). Aerodynamics of Swept and Leaned Transonic CompressorRotors. Applied Energy 84(10), 1012-1027.

Biollo, R. and E. Benini (2009). Shock/BoundaryLayer/Tip-Clearance Interaction in a Transonic Rotor Blade. Journal of Propulsion and Power 25(3), 668-677.

Breugelmans, F. A. E. (1985). Influence of Incidence Angle on the Secondary Flow in Compressor Cascade with Different Dihedral Distribution. Proceedings of 7 th International Symposium on 
Air Breathing Engines, Beijing, China, 663668 .

Cao, Z., B. Liu and T. Zhang (2017). Influence of Coupled Boundary Layer Suction and Bowed Blade on Flow Field and Performance of a Diffusion Cascade. Chinese Journal of Aeronautics 30(1), 249-263.

Cao, Z., X. Gao and B. Liu (2019). Control Mechanisms of Endwall Profiling and its Comparison with Bowed Blading on Flow Field and Performance of a Highly-loaded Compressor Cascade. Aerospace Science and Technology 95, 105472.

Chunill, H. and C. Rabe Douglas (2004). Role of Tip-leakage Vortices and Passage Shock in Stall Inception in a Swept Transonic Compressor Rotor. Proceedings of ASME Turbo Expo 2004: Power for Land, Sea, and Air, Vienna, Austria, GT2004-53867.

Deich, M. E., A. B. Gubalev and G. A. Filippov (1962). A New Method of Profiling the Guide Vane Cascade of Stage with Small Ratios Diameter to Length. Teplienergetika 8(8), 4246.

Denton, J. D. and L. Xu (2002). The Effects of Lean and Sweep on Transonic Fan Performance. Proceedings of ASME Turbo Expo 2002, Amsterdam, The Netherlands, GT-2002-30327.

Du, J., F. Lin and J. Chen (2013). Flow Structures in the Tip Region for a Transonic Compressor Rotor. Journal of Turbomachinery 135(3), 031012 .

Fischer, A., W. Riess and J. R. Seume (2004). Performance of Strongly Bowed Stators in a Four-Stage High-Speed Compressor. Journal of Turbomachinery 126(3), 333-338.

Hofmann, W. and J. Ballmann (2002). Tip Clearance Vortex Development and Shock-Vortex Interaction in a Transonic Axial Compressor Rotor. Proceedings of AIAA Aerospace Sciences Meeting and Exhibit, Reno, NV, AIAA 2002-0083.

Kan, X., W. Wu and J. Zhong (2020). Effects of Vortex Dynamics Mechanism of Blade-end Treatment on the Flow Losses in a Compressor Cascade at Critical Condition. Aerospace Science and Technology 102(7), 105857.

Li, H., Y. Liu and L. Ji (2018). Effect of Nonuniform Tip Clearance on the Performance of Transonic Axial Compressors. Journal of Propulsion and Power, 34(3), 808-818.

Liu, Y., Y. Tang and P.G. Tucker (2020). Modification of Shear Stress Transport Turbulence Model Using Helicity for Predicting Corner Separation Flow in a Linear Compressor Cascade. Journal of Turbomachinery 142(2), 021004 .

Oyama, A., M. Liou and S. Obayashi (2003). Highfidelity Swept and Leaned Rotor Blade Design
Optimization Using Evolutionary Algorithm. 16th AIAA Computational Fluid Dynamics Conference, Orlando, Florida, AIAA 2003-4091.

Perrin, G. and F. Leboeuf (1992). Analysis of Threedimensional Viscous Flow in a Supersonic Axial Flow Compressor Rotor with Emphasis on Tip Leakage Flow. Proceedings of ASME International Gas Turbine and Aeroengine Congress and Exposition, Cologne, Germany, 92-GT-388.

Qiao, B., Y. Ju and C. Zhang (2019). Numerical Investigation on Labyrinth Seal Leakage Flow and Its Effects on Aerodynamic Performance for a Multistage Centrifugal Compressor. Journal of Fluids Engineering 141(7), 071107.

Razavi, S. R. and M. Boroomand (2014). Numerical and Performance Analysis of one row Transonic Rotor with Sweep and Lean angle. Journal of Thermal Science 23(5), 438-445.

Sasaki, T. and F. A. E. Breugelmans (1998). Comparison of Sweep and Dihedral Effects on Compressor Cascade Performance. Journal of Turbomachinery 120(2), 454-464.

Shi, K. and S. Fu (2013). Study of Shock/Blade Tip Leakage Vortex/Boundary Layer Interaction in a Transonic Rotor with IDDES Method. Proceedings of ASME Turbo Expo 2013: Turbine Technical Conference and Exposition, San Antonio, Texas, GT2013-95252.

Suder, K.L. (1996). Experimental Investigation of the Flow Field in a Transonic, Axial Flow Compressor with Respect to the Development of Blockage and Loss. Lewis Research Center, NASA, Cleveland, Ohio.

Sun, S., S, Wang and S. Chen (2019). The Impact of Various Forward Sweep Angles on the Performance of an Ultra-high-load Lowreaction Transonic Compressor Rotor. Applied Thermal Engineering, 150, 953-966.

Sun, S., S. Wang and S. Chen (2020). The Influence of Diversified Forward Sweep Heights on Operating Range and Performance of an Ultrahigh-load Low-reaction Transonic Compressor Rotor. Energy, 194, 116857.

Sun, J., X. Ottavy and Y. Liu (2021). Corner Separation Control by Optimizing Blade End Slots in a Linear Compressor Cascade. Aerospace Science and Technology 114, 106737.

Takahashi, Y., H. Hamatake and Y. Katoh (2005). Experimental and Numerical Investigations of Endwall Flow in a Bowed Compressor Cascade. 41st AIAA/ASME/SAE/ASEE Joint Propulsion Conference and Exhibit, Tucson, Arizona, AIAA 2005-3638.

Tang, Y., Y. Liu and L. Lu (2020). Passive Separation Control with Blade-End Slots in a Highly Loaded Compressor Cascade. AIAA Journal 58(1), 85-97. 
Wang, Z., S. Lai and W. Xu (1981). Aerodynamic Calculation of Turbine Stator Cascades with Curvilinear Leaned Blades and some Experimental Results. Proceedings of Symposium of 5th International Symposium on Air Breathing Engines, Bangalore, India.

Welngold, H. D., R. J. Neubert and R. F. Behlke (1995). Reduction of Compressor Stator Endwall Losses Through the Use of Bowed Stators. Proceedings of ASME International Gas Turbine and Aeroengine Congress and Exposition, Houston, Texas, 95-GT-380.

Welngold, H. D., R. J. Neubert and R. F. Behlke (1997). Bowed Stators: An Example of CFD Applied to Improve Multistage Compressor
Efficiency. Journal of Turbomachinery 119(2), 161-168.

Yamada, K., M. Furukawa and T. Nakano (2004). Unsteady Three-dimensional Flow Phenomena due to Breakdown of Tip Leakage Vortex in a Transonic Axial Compressor Rotor. Proceedings of ASME Turbo Expo 2004: Power for Land, Sea, and Air, Vienna, Austria, GT2004-53745.

Yan, S., W. Chu and Y. Li (2021). Investigation on the Mechanism of Blade Tip Recess Improving the Aerodynamic Performance of Transonic Axial Flow Compressor. Journal of Applied Fluid Mechanics 14(2), 657-668. 